\title{
The relation of form perception to hue and fundus pigmentation
}

\author{
NANCY B. MITCHELL and ROBERT H. POLLACK \\ University of Georgia, Athens, Georgia 30602 \\ and \\ JOHN F. McGREW \\ College of Wooster, Wooster, Ohio 44691
}

\begin{abstract}
A replication of Mitchell and Pollack (1974) has substantiated the relation between color form perception and fundus pigmentation in free viewing task performance. Furthermore, reducing available light induced a comparable performance decrement in lightly pigmented subjects, indicating the peripheral nature of the phenomenon. Performance of the Wechsler-Bellevue block-design subtests, Forms I and II, in both red/white and blue/yellow conditions by all subjects, produced a performance decrement for darkly pigmented subjects in the blue/yellow condition regardless of lighting levels. Lightly pigmented subjects exhibited no performance difference by color with sufficient lighting.
\end{abstract}

Racial differences in sensitivity to the Müller-Lyer illusion have generated two opposing hypotheses. The first suggests that the degree of susceptibility to this illusion is, in part, a function of the subjects visual ecology or the visual environment (Segal, Campbell, $\&$ Herskovits, 1966). The second hypothesis, proposed by Pollack (Pollack \& Silvar, 1967; Silvar \& Pollack, 1967), accounts for these differences in terms of pigmentation within the eye itself, fundus pigmentation. In addition to the supportive evidence for this hypothesis generated by Pollack and his colleagues (Ebert \& Pollack, 1972; Sjostrom \& Pollack, 1971), a number of psychophysical studies (Ishak, 1952a, b; Eckhardt, Note 1; Fitzpatrick, Note 2) have shown that racial differences in fundus pigmentation also seem to account for reduced sensitivity to the short wave lengths of light, primarily to the color blue. For an excellent review of the debate and evidence, see Bornstein (1973).

To what extent do the factors of fundus pigmentation influence visual perception in tasks of an applied nature as opposed to tasks that are purely psychophysical? Mitchell and Pollack (1974) have produced evidence which suggests that differences in occular pigmentation result in differential performance on a block design task which requires color form perception. Their results support those of Jahoda (1971), who used color in a spatial viewing task.

Using the WISC block design subtest to test Pollack's hypothesis in a "real world" task, Mitchell and Pollack (1974) found a performance decrement for black children (4th and 5th graders) making blue and yellow block designs when their performance was compared to another group of black children who made the block designs in red and white. The scores for the two groups were significantly different. When the group of black children who did the task using the red and white blocks was compared to an equivalent sample of white children (for whom design color made no difference), the groups performed equally well. A partial replication (Mitchell \& Pollack, Note 3) and a group of parametric studies (Mitchell \& Pollack, Note 4), in which color lightness or contrast was controlled and saturation was varied, yielded ambiguous results. These results indicated that the available ambient illumination might be an influential factor in performance. The studies were all done in grammar schools in Georgia and Alabama, where standard laboratory conditions were difficult to maintain. Therefore, a complete and thoroughly controlled replication of the original results using adult subjects was felt to be necessary.

The present study, then was done for two reasons: (1) to attempt to replicate and expand the original findings using two controlled levels of lighting, normal and low, and (2) to formulate a valid within-subjects design. Previous results and the fundus pigmentation hypothesis led us to expect that the poorest performance would come from the darkly pigmented subjects forming the designs in blue and yellow under low levels of illumination. We also expected that the within-subject performance for both groups, lightly and darkly pigmented, would exhibit a decrement when the designs were made in blue and yellow as the level of illumination diminished. The peripheral nature (occular as opposed to central or cognitive) of the color-related phenomenon would be 
verified as well if, as we anticipated (Sjostrom \& Pollack, 1971), all subjects experienced difficulty under conditions of low illumination.

\section{METHOD}

\section{Subjects}

The subjects were 16 white and 16 black college freshmen and sophomores from the University of Georgia and the University of Tulsa. Both sexes were equally represented. Subjects at both universities were given course credit for participating.

\section{Procedure}

Each subject did the Wechsler-Bellevue block design subtest, Forms I and II, in red/white and blue/yellow. Order of presentation for form was counterbalanced and color by design form was balanced across subjects. For example, one subject did Form I in blue/yellow and Form II in red/white. For another subject, color and test form were reversed. The standard test materials and instructions were used throughout.

Lighting was provided by four G.E. chroma 75 fluorescent bulbs, $4 \mathrm{ft}$ in length, in a ceiling fixture. The light temperature was $7,500^{\circ} \mathrm{K}$, approximating that of illuminant centigrade or diffuse northern daylight. The testing rooms were painted black. All subjects wore Maiorca swim goggles with lens. For half of the subjects, 1.5 neutral-density filters covered the lens, reducing the available light by $90 \%$.

\section{RESULTS AND DISCUSSION}

The experimental design included four factors: lighting occular pigmentation as determined by race membership, design color, and design form. There were two levels for each factor, with repeated measures on design color. Design form was an incomplete factor. As previous evidence (Barry, Fulkerson, Kubala, \& Seaquist, 1956; Gibbey, 1949) had indicated a high correlation between the two block design forms (Wechsler-Bellevue I and II), equivalence of design form was analyzed by means of a $\mathrm{t}$ test for correlated means. For this comparison $\mathrm{t}=.223$, thus indicating no reliable difference.

The ANOVA performed on the remaining factors showed significant main effects for pigmentation $(\mathrm{p}<.001)$ and for design color $(\mathrm{p}<.001)$. A significant interaction between lighting level, pigmentation, and design color $(\mathrm{p}<.001)$ indicated that an analysis of the simple effects was necessary.

Although lighting level in itself did not produce a significant main effect, the simple effects of lighting at the level of pigmentation suggested that the level of illumination had a greater effect on the darkly pigmented subjects $(F=2.478)$ than on the lightly pigmented subjects $(F=.694)$. The greatest effect of lighting on color was at the lower level of illumination $(F=20.06, p<.001)$, and the greatest effect of color was evident for darkly pigmented subjects $(\mathrm{F}=15.46, \mathrm{p}<.001)$. At both levels of pigmentation, blue and yellow blocks produced the greater performance deficit $(\mathrm{F}=81.17, \mathrm{p}<.001)$.

A Tukey multiple comparison test on the means indicated that blue/yellow design performance produced significantly $(p<.01)$ lower scores at low levels of illumination than scores produced by designs done in red/white. For darkly pigmented subjects, means for blue/yellow performance were significantly lower than means for red/white performance $(p<.01)$. Because of the significant interaction, more specific comparisons could not be made, but an illustration of the results (Figure 1) indicates that the predictions were confirmed.

The comparison of explicit interest was the within-subjects performance scores for darkly pigmented subjects wearing goggles with filters. While the specific comparison cannot be made, it is apparent from Figure 1 that the difference between the mean score for blue/yellow performance compared to red/white performance is greater for subjects wearing goggles with filters. When levels of illumination are pooled, blue/yellow design performance was poorer than red/white design performance $(p<.01)$. For lightly pigmented subjects, blue/yellow design performance was poorer than red/white design performance when filters were worn. There was no difference between the means as a function of design color when filters were not worn. The lack of difference between scores as a function of design color when illumination is normal confirms earlier findings. The difference in scores as a function of design color which exists for lightly pigmented subjects wearing filters is a good indication that the phenomenon is mainly peripheral in nature, since it can be induced under proper conditions of illumination independent of central cognitive factors. It is interesting to note that in the low-illumination condition all scores

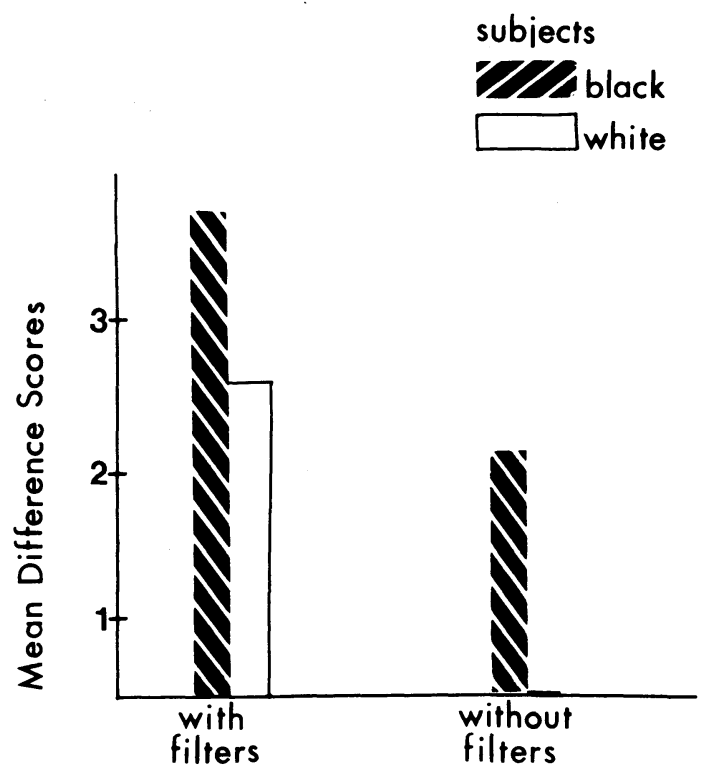

Figure 1. Mean difference scores between performance on the $\mathrm{red} /$ white and blue/yellow versions of the Wechsler-Bellevue block design subtest. Red/white scores are superior to blue/yellow scores. 
produced by doing the designs in blue/yellow were lower than all scores produced by making the design in red/white.

In general, the results of this study confirm in adults the results originally obtained with children (Mitchell \& Pollack, 1974). The more precise specification of illumination and the use of the neutral-density filter to reduce the level of illumination make clearer the subtle interaction between light intensity and the effects of fundus pigmentation. The density of occular pigmentation in the black subjects under normal levels of illumination appears to lower their sensitivity to blue sufficiently to interfere with the accurate perception of the forms produced by blue-yellow contrast. This interference is sufficient to produce a significant decrement in the performance of black subjects using these blocks. One very astute, young, black man, at the end of the testing session announced that he knew what the experiment was about. He said that he could not see the blue and yellow designs as well as he saw the red and white designs. The imposition of the neutraldensity filter not only causes a further decrement in the performance of the black subjects in this condition but also appears to simulate an increase in the density of fundus pigmentation in the white subjects. This finding makes it clear that we are dealing with a peripheral optical effect based upon the interaction of fundus pigmentation and illumination intensity. not with a cognitive effect related in any way to the social factors inherent in race differences.

What the long-term theoretical effects of the interaction between fundus pigmentation and color form perception might be, we have no way of knowing at present, except to say that it does appear to affect performance on tasks which are based upon form perception produced by color contrast. This interaction, which seems especially pronounced for the complementary colors, yellow and blue (Bornstein, 1973), may have theoretical implication for the central processing of visual information (Dow \& Gouras, 1973) which have not yet been investigated. The results of this series of experiments must certainly be considered in interpreting data from cross-cultural studies which make demands on form perception determined by color contrasts.

\section{REFERENCE NOTES}

1. Eckhardt, R. A. Foveal luminosity functions in relation to macular and skin pigmentation. Unpublished doctoral dissertation, Fordham University, 1966.
2. Fitzpatrick. G. E. An investigation of the role of macular pigmentation in spectral sensitivity to the short wave lengths. Unpublished doctoral dissertation, Fordham University, 1974.

3. Mitchell, N. B.. \& Pollack, R. H. Block design performance as a function of race and block design color. Paper presented at the biennial meeting of the Society for Research in Child Development, Philadelphia, March 1973.

4. Mitchell. N. B.. \& Pollack, R. H. The effects of saturation on block design performance in black children. Paper presented at the annual meeting of the Eastern Psychological Meeting. Philadelphia. April 1974.

\section{REFERENCES}

Barry, J. R., Fulkerson, S. C., Kubala. A. L., \& Seaquist, M. R. Score equivalence of the Wechsler-Bellevue intelligence scales, forms I and II. Journal of Clinical Psychology, 1956, 12, 57-60.

Bornstein, M. H. The psychophysiological component of cultural differences in color naming and illusion susceptibility. Behavior Science Notes, 1973, 8, 41-101. Human relations area files, 2054 Yale Station. New Haven. Conn. 06520.

Dow. B. M.. \& Gouras, P. Color and spatial specificity of single units in Rhesus monkey foveal striate cortex. Journal of Neurophysiology, 1973, 36. 79-100.

Ebert, P. C., \& Pollack, R. H. Magnitude of the MuellerLyer illusion as a function of hue, saturation, and fundus pigmentation. Psychonomic Science, 1972, 26, 225-226.

GiB BY. R. G. A preliminary survey of certain aspects of form II of the Wechsler-Bellevue as compared to form I. Journal of Clinical Psychology, 1949, 5, 165-169.

IsHaK. I. G. H. The photopic luminosity curve for a group of tifteen Egyptian trichromats. Journal of the Optometric Society of America, 1952. 42. 534-540. (a)

IsнAK. I. G. H. The spectral chromaticity coordinates for one British observer and eight Egyptian trichromats. Journal of the Optometric Society of America, 1952, 42, 529-534. (b)

JAHODA, G. Retinal pigmentation, illusion susceptibility, and space perception. International Journal of Psychology, 1971, 6, 199-208.

Mitchell, N. B., \& Pollack, R. H. Block-design performance as a function of hue and race. Joumal of Experimental Child Psychology, 1974, 17, 377-382.

Pollack. R. H.. \& Silvar, S. D. Magnitude of the MuellerLyer illusion in children as a function of pigmentation of the fundus oculi. Psychonomic Science, 1967, 8, 83-84.

Segal, M. H., Campbell, E. F., \& Herskovits, M. J. Influence of culture on visual perception. Indianapolis: Bobbs Merril. 1966.

Silvar, S. D., \& Pollack, R. H. Racial differences in pigmentation of the fundus oculi. Psychonomic Science. 1967 . 7. 159-160.

Siostrom. K.. \& Pollack, R. H. The effect of simulated receptor aging on two types of visual illusions. Psychonomic Sicince. 1971. 23, 147-148. 